\title{
Genetic architecture of paediatric renal diseases in China and the need for data sharing
}

\author{
Agnieszka Bierzynska ${ }^{1}$, Moin A. Saleem ${ }^{1,2}$ \\ ${ }^{1}$ Bristol Renal, Translational Health Sciences, Bristol Medical School, University of Bristol, Bristol, UK; ${ }^{2}$ Bristol Royal Hospital for Children, \\ Bristol, UK \\ Correspondence to: Dr. Agnieszka Bierzynska. Bristol Renal, Translational Health Sciences, University of Bristol, Dorothy Hodgkin Building, Whitson \\ Street, Bristol BS1 3NY, UK. Email: A.Bierzynska@bristol.ac.uk. \\ Provenance and Peer Review: This article was commissioned by the editorial office, Translational Pediatrics. The article did not undergo external peer \\ review. \\ Comment on: Rao J, Liu X, Mao J, et al. Genetic spectrum of renal disease for 1001 Chinese children based on a multicenter registration system. Clin \\ Genet 2019;96:402-10.
}

Submitted May 01, 2020. Accepted for publication May 22, 2020.

doi: $10.21037 /$ tp-20-135

View this article at: http://dx.doi.org/10.21037/tp-20-135

Next generation sequencing (NGS), which became widely used during the last decade, has enabled incredible progress in genetic diagnostics of many inherited diseases. In the era of precision medicine, NGS is considered a gold standard for the diagnosis of Mendelian diseases thanks to its high throughput, cost effectiveness and high accuracy. This opens avenues for personalised medicine, including tailored treatment strategies and disease prognosis.

A recent paper published by J Rao, X Liu, J Mao and colleagues for "Internet Plus" Nephrology Alliance of National Center for Children's Care presents a genetic spectrum of renal diseases in a national cohort of 1,001 Chinese children. Patients were recruited to the Chinese Children Genetic Kidney Disease Database (CCGKDD), which is the largest national multicentre registration system for the genetic diagnosis of renal disease. Little is known about the genetic spectrum of renal disease in paediatric patients in China. Thus, the CCGKDD cohort provides a unique opportunity to establish the mutation rate in young renal patients in this country. Around 30\% of chronic kidney disease (CKD), with onset $<25$ years, can be attributed to a genetic cause. There is, however, a substantial variability between the reported frequency of monogenic cases depending on the underlying phenotypes included in the studies (1). The four most common diagnostic groups for early onset ( $<25$ years) CKD are congenital anomalies of the kidneys and urinary tract (CAKUT), steroid resistant nephrotic syndrome (SRNS), chronic glomerulonephritis and renal cystic ciliopathies. Two thirds of early-onset CKD are due to those 4 subgroups and over 200 genes have been found to cause early onset CKD when mutated (2).

In the CCGKDD cohort, genetic diagnosis was confirmed in $42.1 \%$ of patients with $60.7 \%$ of those having a causative mutation in 1 of 15 the commonest genes. The authors highlight the fact that overall, in this cohort, mutations were found in 106 genes. As expected, different diagnostic yield was found in different categories of renal disease, i.e., $29.1 \%$ of SRNS were found to be monogenic while in CAKUT $17 \%$ had a mutation. All significant variants authors included in the supplemental material.

Median genetic diagnostic yield for patients with CAKUT $<18$ years is $17 \%$ (1), which is not detected based on standard clinical evaluation. Patients having an unsuspected genetic disorder may be at risk of neurocognitive impairment and thus early recognition of the causative mutation can impact clinical care. Patients with CAKUT have variable phenotype and the disease may also occur in conjunction with defects in other organs thus anatomical classification is often ambiguous with regard to the primary molecular aetiology. At least 50 genes have been found mutated in CAKUT and when a mutation is found is certain genes, e.g., HNF1B and PAX2 screening patients for diabetes or ocular coloboma respectively may be advisory (3). The complex genetic basis of CAKUT 
requires large-scale human genetic studies accompanied with detailed phenotypic information. Therefore, studies like this one by Rao et al., where $17 \%$ of CAKUT patients were found to have a genetic cause, significantly contribute to the understanding the genetic architecture and mutation spectrum of CAKUT.

Studies like this, highlight the differences in geographic distribution of patients with early onset ( $<2$ years) of renal disease. In the case of this study, the proportion of younger patients was higher in Western and Central China than from the Eastern part of the country. This paper also presents the most frequent mutations found in each disease subgroup which emphasises the different allelic distribution in different populations. In SRNS for example, the most frequently mutated genes were COQ8B (also known as ADCK4), WT1 and NPHS1 rather than NPHS1, WT1 and NPHS2 seen by other groups $(4,5)$. Interethnic differences are likely to influence the incidence of mutations in different genes. This group has also found a three times higher than expected incidence of mutations in NPHP3 gene, causing nephronophthisis. Mutations in this gene have been reported in patients with infantile and adolescent-onset but more frequently in the first one $(6,7)$. It is therefore possible that the higher mutation rate may be influenced by the number of patients with early onset of the disease recruited to this study, however the average onset for the nephronophthisis patients is not provided. Establishing molecular genetic diagnosis can be particularly important in patients requiring renal transplant. It is known that patients with monogenic form of SRNS are less likely to have a disease recurrence post-transplant (4). However, this is not the case for all "nephrotic" genes and/or mutations. Congenital nephrotic syndrome of the Finnish type is caused by mutations in NPHS1. The majority of these patients have a homozygous truncating mutation (Fin-major) which results in the lack of protein nephrin and anti-nephrin antibodies are developed in these patients after the transplant leading to disease recurrence in the "new kidney" (8). Very rarely post-transplant recurrence has also been noted in patients with NPHS2, WT1 or NUP93 mutations $(9,10)$. Genetic diagnosis before transplant is also extremely important when a related donor is considered to avoid using a kidney from an affected member who may not yet show the symptoms.

Due to the lack of treatment, SRNS is one of the most challenging renal conditions. However, in some patients supplemental medication may be possible. It is known that when SRNS it is caused by mutations in certain genes, additional treatment options seem beneficial. In this cohort, one of the most commonly mutated genes in the SRNS patients was COQ8B. COQ8B is involved in CoQ10 biosynthesis and patients with mutations in this gene may benefit from coenzyme Q10 supplementation (11). It has been shown that, if applied early, oral supplementation of CoQ10 may not only reduce proteinuria but also stabilize kidney function in COQ nephropathies (11-13). In some patients with mutations in podocyte genes partial or complete remission may be possible when treated with cyclosporine A (14).

Genetic studies on large cohorts help us to understand what mutations are the most frequent in different populations and sharing the data with others strengthens precise mutation classification. Therefore, data from this cohort is extremely valuable and helps us to better understand the genetic architecture of early onset kidney diseases in China. Detailed phenotypic information as well as follow-up information are also vital for correct genotype-phenotype correlations. One of the limitations of this work, noted by the authors, was that they were unable to investigate the details of phenotype and follow up information. The authors stressed that improving the phenotype on a genomic scale needs to be included in the future work on CCGKDD. Even though, the present cohort was not a population-based cohort of Chinese children, and it is possible that more severe cases were sent to the medical centres involved in this study, still this work sheds light on the genetic spectrum of paediatric renal disease in China.

Three approaches for genetic testing were applied in this study. Targeted gene sequencing (TGS) where a large gene panel with 2,703 genes was used, including 263 known genes responsible for kidney disease and thus covering $73.1 \%$ of the priority nephropathy genes. Second method used was whole exome sequencing (WES) which was available in parallel but was performed in more complex cases like SRNS, CAKUT or CKD with unknown origin as well as patients where mutation was not found by TGS. The third approach used was trioWES which was found to be extremely helpful especially in renal tubular disease and renal calcinosis patients enabling significantly higher mutation detection rate compared to the first two approaches. Studies often suffer from having many variants of unknown significance (VUS), mainly due to the lack of parental DNA and thus not being able to confirm trans character or de novo status of a mutation. It is therefore somehow surprising that trio-WES didn't 
increase the genetic diagnostic rate in other diseases in this study. This could potentially be caused by differences in patient selection for different approaches. The authors acknowledge that TGS was favoured in certain patients, including infantile or more severe nephrotic syndrome where mutation rate is likely to be higher than in later onset or milder disease.

WES and trio-WES data have a great potential for further work as it should be possible to look for novel gene candidates for paediatric kidney disease. It is however not clear whether the authors have any plans to investigate this, even for familial cases where no causative mutation was found in the known genes. Utilising WES data is also beneficial to detect mutations in genes that were not originally suspected from the clinical phenotype due to overlapping phenotypes/phenocopy genes. For some patients correct diagnosis may not be possible without checking other genes since multiple genes often contribute to a specific phenotype and/or single gene defects have multiple clinical presentations. For instance, mutations in SLC4A1 and AGXT (known to cause distal renal tubular acidosis and hyperoxaluria type 1 respectively) were found in patients with renal cystic ciliopathies instead of typical/ known genes for this condition (2). Similarly, we found an OCRL mutation in a patient where clinical and renal biopsy findings indicated glomerular disease while his brother received presumptive diagnosis of IgA nephropathy. The typical for $O C R L$ gene mutations, Lowe syndrome or Dent disease, were not suspected in the siblings' clinical presentation $(4,15)$.

Copy number variation (CNV) analysis is known to be challenging in WES. Deletion in NPHP1 gene, detected in several patients was further validated with other approaches, e.g., real-time PCR. Authors appreciate the limitation for $\mathrm{CNV}$ detection in this study and stress that some patients, where no pathogenic variant was detected, were referred for further whole genome sequencing.

Data sharing is important as it helps understand the disease and its mechanisms better. The same (or similar) phenotype can be caused by different genetic mutations and vice versa the same gene/mutation may be responsible for different phenotypes. Thus, sharing combined genotype and phenotype data is extremely important and initiatives like patient registries/national cohorts are pivotal in gaining knowledge about complex genetic disorders. Establishment of the CCGKDD further aids our knowledge and provides a great resource of mutations detected in China.

\section{Acknowledgments}

The authors would like to acknowledge support from Kidney Research UK. A.B.

Funding: This work is funded by Kidney Research UK (Non-clinical Post-doctoral Fellowship).

\section{Footnote}

Conflicts of Interest: Both authors have completed the ICMJE uniform disclosure form (available at http://dx.doi. org/10.21037/tp-20-135). MAS reports personal fees from Retrophin, outside the submitted work. $\mathrm{AB}$ has no conflicts of interest to declare.

Ethical Statement: The authors are accountable for all aspects of the work in ensuring that questions related to the accuracy or integrity of any part of the work are appropriately investigated and resolved.

Open Access Statement: This is an Open Access article distributed in accordance with the Creative Commons Attribution-NonCommercial-NoDerivs 4.0 International License (CC BY-NC-ND 4.0), which permits the noncommercial replication and distribution of the article with the strict proviso that no changes or edits are made and the original work is properly cited (including links to both the formal publication through the relevant DOI and the license). See: https://creativecommons.org/licenses/by-nc-nd/4.0/.

\section{References}

1. Connaughton DM, Hildebrandt F. Personalized medicine in chronic kidney disease by detection of monogenic mutations. Nephrol Dial Transplant 2020;35:390-7.

2. Vivante A, Hildebrandt F. Exploring the genetic basis of early-onset chronic kidney disease. Nat Rev Nephrol 2016;12:133-46.

3. Sanna-Cherchi S, Westland R, Ghiggeri GM, et al. Genetic basis of human congenital anomalies of the kidney and urinary tract. J Clin Invest 2018;128:4-15.

4. Bierzynska A, McCarthy HJ, Soderquest K, et al. Genomic and clinical profiling of a national nephrotic syndrome cohort advocates a precision medicine approach to disease management. Kidney Int 2017;91:937-47.

5. Sadowski CE, Lovric S, Ashraf S, et al. A single-gene cause in $29.5 \%$ of cases of steroid-resistant nephrotic syndrome. J Am Soc Nephrol 2015;26:1279-89. 
6. König J, Kranz B, Konig S, et al. Phenotypic Spectrum of Children with Nephronophthisis and Related Ciliopathies. Clin J Am Soc Nephrol 2017;12:1974-83.

7. Sun L, Tong H, Wang H, et al. High mutation rate of NPHP3 in 18 Chinese infantile nephronophthisis patients. Nephrology (Carlton) 2016;21:209-16.

8. Holmberg C, Jalanko H. Congenital nephrotic syndrome and recurrence of proteinuria after renal transplantation. Pediatr Nephrol 2014;29:2309-17.

9. Bierzynska A, Saleem MA. Deriving and understanding the risk of post-transplant recurrence of nephrotic syndrome in the light of current molecular and genetic advances. Pediatr Nephrol 2018;33:2027-35.

10. Seeman T, Vondrak K. First Report of Recurrent Nephrotic Syndrome After Kidney Transplantation in a Patient With NUP93 Gene Mutations: A Case Report.

Cite this article as: Bierzynska A, Saleem MA. Genetic architecture of paediatric renal diseases in China and the need for data sharing. Transl Pediatr 2020;9(3):202-205. doi: $10.21037 /$ tp-20-135
Transplant Proc 2018;50:3954-6.

11. Ashraf S, Gee HY, Woerner S, et al. ADCK4 mutations promote steroid-resistant nephrotic syndrome through CoQ10 biosynthesis disruption. J Clin Invest 2013;123:5179-89.

12. Atmaca M, Gulhan B, Korkmaz E, et al. Follow-up results of patients with ADCK4 mutations and the efficacy of CoQ10 treatment. Pediatr Nephrol 2017;32:1369-75.

13. Ozaltin F. Primary coenzyme Q10 (CoQ 10) deficiencies and related nephropathies. Pediatr Nephrol 2014;29:961-9.

14. Kemper MJ, Lemke A. Treatment of Genetic Forms of Nephrotic Syndrome. Front Pediatr 2018;6:72.

15. Preston R, Naylor RW, Stewart G, et al. A role for OCRL in glomerular function and disease. Pediatr Nephrol 2020;35:641-8. 